\title{
Espace et parcours identitaires de la femme antillaise dans La migration des coeurs de Maryse Condé
}

\author{
Maria Anagnostopoulou-Hielscher \\ Rice University, Houston
}

Dans son article "Private Selves and Public Spaces: Autobiography and the African Woman Writer," Carole Boyce Davies explore les difficultés affrontées par la femme noire aussitôt qu'elle décide de se lancer dans l'entreprise autobiographique. Doublement dominée par le pouvoir patriarcal et colonial qui la reléguèrent jusqu'alors au silence et à l'invisibilité, elle doit en effet se montrer capable de se déplacer des marges de la culture vers le centre et assumer ainsi une narration qui depuis toujours définit le sujet comme mâle. Maryse Condé figure parmi une minorité d'écrivains post-coloniaux qui célèbrent ouvertement, par le bias de leurs créations littéraires, l'entrée féminine dans cet espace historique et social qui lui défendit jadis d'exprimer librement son individualité.

Cette étude se concentrera sur un des ouvrages les plus récents de cet auteur guadeloupéen, intitulé La migration des coeurs. Inspiré du chef-d'oeuvre d'Emily Brontë, Wuthering Heights, ce roman traite de la période qui succéda à l'abolition de l'esclavage. Il retrace la vie d'une jeune mulâtresse, Cathy qui, après avoir trahi son ami d'enfance Razyé à cause de sa couleur noire et de son manque d'éducation, épouse un planteur riche, Aymeric de Linsseuil. Marié à Irmine, la soeur d'Aymeric, Razyé se vengera de son amour perdu sur tous ceux qu'il juge responsables de son malheur.

Ce texte nous offre un exemple éloquent d'une a "autobiographie plurielle," à travers laquelle se développe une histoire non seulement personnelle mais aussi collective, narrée par une multitude de voix marginales, féminines pour la plupart, qui, mêlées à celle de l'auteur, se complètent les unes les autres. La polyphonie narrative enrichit la portée du message narratif, contenu dans ce genre de textes que Philippe Lejeune appela référentiels:

Par opposition à toutes les formes de fiction, la biographie et l'autobiographie sont des textes référentiels: exactement comme le discours scientifique ou historique, ils prétendent apporter une information sur une 'réalité' extérieure au texte, et donc se 
soumettre à une épreuve de vérification. Leur but n'est pas la simple vraisemblance, mais la ressemblance au vrai. Non l' 'effet du réel,' mais l'image du réel. (36)

Qu'elle soit religieuse, socio-politique ou historique, cette image du réel paraît à première vue obéir à un binarisme explicite qui sépare deux mondes hétérogènes, fonctionnant uniquement selon la dialectique hégélienne du Maître et de l'Esclave. Dans son essai'The Other Question: Difference, Discrimination and the Discourse of Colonialism," Homni K. Bhabha souscrit aux propositions déjà formulées par les écrivains anti-coloniaux, qui conçoivent le colonisé comme une réalité fixe, négative, aliénante, inauthentique et par ce fait diamétralement opposée à celle représentée par le colon (154). Néanmoins, Bhabha réussit à investir son argument d'une portée encore plus significative, lorsqu'il rejette dans ces écrits la notion d'une relation symétrique entre le colon et le colonisé. ${ }^{1} \mathrm{~L}$ 'exercice du pouvoir colonial est caractérisé, d'après lui, par une ambivalence fondamentale. Alors que l'Occident souligne l'importance de sa mission civilisatrice et sa volonté de créer un Autre qui lui ressemble, il se présente en même temps comme une force violente d'assujettissement et dévoile par ses actions son désir primordial de rester le Maître et de continuer ainsi à contrôler tous ceux qu'il assure protéger. Son projet n'est donc pas de former un double de lui-même mais de "camoufler" ses voeux d'expansion et de conquête tout en faisant étalage de sa suprématie et de son savoir. Voilà pourquoi, lorsque le colonisé essaie d'imiter les manières et le comportement de ses Maîtres, on a l'impression d'assister à une parodie, une dramatisation qui ne reflète guère l'exercice des relations coloniales dépendantes à travers une identification narcissique ("Of Mimicry..." 125-33).

L'ambivalence du pouvoir colonial se manifeste dès que l'on pénètre le sanctuaire même du colon, à savoir l'habitation. Lieu par excellence "d'exils culturels," vu qu'elle fut peuplée non seulement par de "Africains déportés," mais aussi par des "Européens en exil" et parfois "en rupture de ban" (LC 37), ${ }^{2}$ celle-ci proposa une des illustrations les plus convaincantes de ce que fut la culture créole. Il n'en demeure pas moins que pendant l'esclavage et même après, elle ne cessa de hanter l'imagination populaire. Associée à un espace hostile et dangereux, elle fut un appel constant de la présence coloniale et finit par acquérir toutes les propriétés attribuées à la demeure d'un Dieu, patriarche terrifiant, jaloux et distant, dont le portrait s'apparente à celui qu'on trouve dans l'Ancien Testament. Ce parallélisme entre l'habitation blanche et l'Eglise chrétienne n'est nullement exagéré, notamment si l'on se fie à la pensée de Frantz Fanon. Ce dernier nous informe dans son livre Peau noire, masques blancs que "le Dieu bon et miséricordieux [...] est un Blanc quia des joues bien roses" (41), et qu'il ne peut par conséquent résider que dans le bastion de ceux sur qui il veille.

Perçu comme une transgression des lois immuables prescrites par le chef de cette Eglise qui "appelle [...] l'homme colonisé [...] dans la voie de l'oppresseur" (DT 72), ${ }^{3}$ le mariage chrétien de Cathy, narré par son frère Justin, consacre son passage officiel au monde de la blancheur: 
Les deux semaines qui ont précédé le mariage, il a plu à verse [...] Le ciel était pareil à un soulard qui pissait [...] sans jamais se vider entièrement. [...] Devant cette étrangeté, les gens [...] disaient que s'il pleuvait pareillement, c'est que l'eau des pleurs serait au rendez-vous de cette noce qui se célébrait dans la soie et l'or. [...] Cependant, ô miracle, le 13 avril, le soleil se leva tellement [...] éclatant au-dessus de la mer que depuis huit heures du matin on ne pouvait déjà plus soutenir son regard. Il creva les yeux de deux pêcheurs qui avaient eu le toupet de lever la tête vers lui et brûla les paupières de quelques gamins qui avaient fait de même. (MC 54-55)

La violence des éléments naturels atteste la colère du Tout Puissant envers cette créature insignifiante qui ignora ses avertissements et se permit de réclamer une place de prédilection dans son royaume sacré. Le déluge qui annonce la cérémonie nuptiale est en même temps annonciateur de la dévastation qui accompagnera Cathy pendant le reste de sa vie. Quant à l'éclat solaire, puissance maléfique (Durand 168) qui appelle la destruction, il incarne ici un effort suprême de la part du Roi-Soleil, Dieu et Colon à la fois, de dévorer ceux qui osent le défier.

Si l'on adopte une analyse fanonienne, on pourrait tout simplement expliquer les noces de Cathy avec Aymeric de Linsseuil, si blanc et fragile qu'il est surnommé "chérubin céleste," comme le seul résultat d'un désir impérieux de la part d'une jeune mulâtresse d'améliorer son sort et de gravir l'échelle sociale, malgré tous les sacrifices personnels qu'elle doit endurer. Quoiqu'une explication de la sorte ne soit pas du tout illogique, elle nous paraît assez limitée, car elle ne tient pas compte des complications qu'un tel acte serait susceptible de révéler au niveau des liens qui se tissent entre le Dieu / Colon et le colonisé. Ni noire ni blanche, Cathy rappelle par son statut social ce proverbe cité par Pierre André Taguieff et qu'on retrouvait bien souvent dans les textes qui condamnaient le métissage: "Dieu a créé le Blanc, Dieu a créé le Noir. Le Diable a créé le Métis" (57). Elle ne peut donc qu'occuper la place de l'objet d'abjection, place qui est commentée par Kristeva dans son ouvrage critique Pouvoirs de l'horreur. Ce qui rend abject, nous dit-elle, c'est "ce qui perturbe une identité, un système, un ordre. Ce qui ne respecte pas les limites, les places, les règles. L'entre-deux, l'ambigu, le mixte" (12) Ainsi la protagoniste de La migration des coeurs propose-t-elle une illustration convaincante du terme "métis," tel qu'il est expliqué par Marcel Detienne et Jean-Pierre Vernant:

[...] la métis apparaît [...] multiple, bigarrée, ondoyante [...] [p]arce qu'elle a pour champ d'application le monde du mouvant, du multiple, de l'ambigu. Elle porte sur des réalités fluides, qui ne cessent jamais de se modifier et qui réunissent en elles, à chaque moment, des aspects contraires, des forces opposées. [...] La métis est [...] puissance de ruse et de tromperie. Elle agit par déguisement. Pour duper sa victime elle emprunte une forme qui masque au lieu de révéler, son être véritable. (28-29) 
Cathy était d'après ses mabos "autoritaire, violente, toujours prête à répliquer, sournoise" (MC 26), "éffrontée, mal éduquée et sans manières" (74) sans que cela l'empêche de se montrer aussi babillarde" et "attendrissante" (76). Heureuse tout au long de son enfance, elle accepte la proposition de mariage de Linsseuil, car comme elle le dit à sa servante Nelly, elle ne pourrait jamais se marier avec Razyé: "Ce serait une dégradation! Ce serait comme s'il n'y avait qu'une seule Cathy, la bossale, la mécréante descendant tout droit de son négrier" (48).

Sa transformation étonne son entourage et témoigne de sa volonté d'adopter les valeurs de ce milieu aristocratique dont elle souhaite être acceptée: "Sa bouche était en cul de poule. [...] elle glissait sur ses pieds toujours enfermés dans des chaussures. Elle avait relevé et roulé en chignon ses grands cheveux noirs [...] A présent, elle se souciait de sa couleur, s'abritait d'une ombrelle et recherchait l'ombrage. Au lieu de sauter sur le dos d'un cheval et de galoper dans le soleil, elle s'asseyait sur la galerie et tournait les pages d'un livre" (43). Cependant, son effort de jouer un rôle qui est loin de convenir à son tempérament exubérant et de satisfaire ses besoins émotionnels la plonge dans une crise identitaire qui la condamne à une apathie paradoxale s'accordant mal avec sa personnalité vibrante. Sa vraie nature transparaît lorsqu'elle se met à réfléchir sur sa religion. Une des rares fois où elle assume la narration, elle confie à sa mabo Lucinda ses pensées les plus intimes et revendique pour la première fois son héritage africain:

Je rêve d'un au-delà où nous pourrions exprimer tous les sentiments, toutes les envies que nous avons dû étouffer pendant notre existence. [...] Tu vois, depuis petite, je me demande si la religion chrétienne n'est pas une religion des Blancs faite pour les Blancs; si elle est bonne pour nous autres, qui avons le sang d'Africain dans les veines. [...] La religion qu'on nous enseigne dans les églises interdit tout ce qui donne du sel à l'existence. A cause d'elle, les besoins de notre corps se transforment en malédictions. (86)

Rejetée à jamais par ce Dieu qui nie sa nature humaine, Cathy est en proie à un malaise profond. Celui-ci se manifeste aussi chez Irmine, la soeur d'Aymeric de Linsseuil. Après avoir renoncé à ses privilèges de femme blanche et épousé Razyé, elle se voit abandonnée par ce Dieu dont elle fut autrefois une des enfants les plus choyées: "[...] elle se reprochait de psalmodier ces paroles vides, de respecter ces pratiques dans lesquelles elle n'avait plus de foi. Car elle ne le portait pas dans son coeur, ce bon Dieu tellement mal nommé qui gâtait les uns, anéantissait les autres, qui partageait si inégalement le bonheur et la fortune" (273). Cathy, elle-même, va jusqu'à inventer une contre-prière dont le contenu ironique et irrespectueux nous est révélé par Razyé: 'Nous te haïssons, toi qu'on ne voit jamais, mais qui es assis là-haut dans le ciel. Tu partages sans justice la couleur, les habitations, les terres. Nous ne t'appellerons jamais notre père parce que tu ne l'es pas" (121). Sinistres et désabusés, les deux amis d'enfance ne sont malgré tout pas capables de découvrir une foi qui les satisfait. Les prêtres 
délégués des traditions religieuses venues d'Afrique, vers qui il se tourne, se révèlent être aussi charlatans que ceux qui prêchent le christianisme. Toute voie spirituelle qui pourrait apporter la consolation et apaiser l'âme humaine semble être à jamais fermée. Ayant hérité la peau sombre de son vrai père Razyé, la fille de Cathy, qui porte le même nom que sa mère, est regardée d'une façon méprisable à l'église par le reste des fidèles. Bien qu'une fois à la Désirade la religion chrétienne soit pour elle un refuge contre sa solitude, elle est gênée devant l'hypocrisie des prêtres anglais de la commune de Rosear: "Ils avaient le nez aussi rouge que les oreilles et passaient sans vergogne de la table de la communion à celle des casinos ou à l'arène d'un pitt. Quand le combat de coqs n'était pas fini, ils ne se gênaient pas pour retarder le commencement de la messe" (302). De son côté, la servante indienne des de Linsseuil, Sanjatta, fait preuve d'une confusion grandissante lorsqu'elle se demande quelle est la raison des calamités dont't souffre le pays entier et qu'elle conclut dans un premier temps que les vrais coupables sont les nègres: "[...] les prêtres en chaîne suppliaient les nègres et les mulâtres de se repentir. Pourquoi persécutaient-ils les Blancs-pays? Pourquoi volaient-ils leur bétail et mettaient-ils le feu à leurs cannes! A cause de cela, le bon Dieu n'était plus bon, et sa colère brûlait le pays" (161). Ne comprenant pas pourquoi elle put provoquer elle-même le courroux de Dieu qui permit la mort subite de ses jeunes enfants atteints d'une fièvre typhoïde, elle arrache dans son désarroi toutes les images "du Bon-Pasteur, du Sacré-Coeur de Jésus et de Sainte Vierge Marie" et les remplace par celles "de Kalimaï, de Vinayagar, dieu à tête d'éléphant, et du dieu-singe Hanouman" (161). Elle s'aperçoit cependant avec effroi que les emblèmes de sa religion d'origine la troublent bien davantage et finit également par s'en débarrasser.

Lieu de contradictions internes, l'espace religieux occupé par le Dieu / Colon est envahi par le colonisé et ses propres pratiques confuses et presque oubliées qui continuent pourtant à attester leur présence. Des chemins divergents que s'entrelacent, sans pouvoir se rencontrer, esquissent les contours d'un labyrinthe sans sortie, au milieu duquel la maison coloniale, toujours vulnérable, mais aussi imposants, est prête à engloutir ceux qui osent la braver.

C'est paradoxalement lors de la veillée mortuaire de son corps, que Cathy prend la parole pour avouer l'effacement progressif de son identité dont elle souffrit dans l'enclos hostile de l'habitation. L'indice le plus significatif de son incarcération morale et corporelle semble avoir été ce corset "objet surprenant avec ses baleines et ses tigres en fer [...] [qui] symbolisait [...] le commencement de la fin" (97) et qu'elle acheta avec Justin quand elle commença à fréquenter les de Linsseuil. Son opulence ne lui permet qu'un bref interlude de bonheur, rapidement gâché par la lucidité de son sort: "les jours de bal, quand je regardais dans les glaces ma figure peinturlurée, des boucles à mes oreilles, des chaînes autour de mon cou, je savais que derrière ce masque se cachaient la solitude et les regrets" (97). Quoique la peinture caricaturale de ce que fut sa vie une fois qu'elle arrive à obtenir le statut aristocratique n'a rien de réconfortant en soi, son témoignage constitue par ailleurs un document précieux qui démolit le mythe d'une société blanche infaillible, mystérieuse et solennelle dans laquelle on ne peut 


\section{pas s'infiltrer.}

A leur tour, lorsque les servantes noires des Linsseuil prennent la parole, ce qui est censé être le monopole des Maîtres, leur récit loin d'être une simple énumération d'événements, reconstruit l'histoire antillaise, histoire de l'esclavage, mais aussi des révoltes, de l'héroïsme et de la détermination de tout un peuple qui n'a jamais voulu accepter ses fers. Leurs voix "marginales" parodient celles de leurs patrons, et se font entendre aussi clairement qu'elles.

Sandrine, une des mabos de La migration des coeurs, dévoile un aspect intéressant de la colonisation, quand elle trace, ne fut-ce que brièvement, le portrait de son patron Aymeric de Linsseuil. Celui-ci était un bon maître, lui apprit à lire et à écrire et déposait chaque mois sa paie à la banque. Les renseignements qu'elle fournit s'accordent avec ceux qu'on trouve dans le reste du roman et complètent son portrait. On apprend qu'il avait fait construire dans son domaine une école, qu'il avait fait réparer et repeint les cages des nègres et qu'il avait été le premier à faire installer des moulins à vapeur. Il rêvait de transformer "Belles-Feuilles en une plantation modèle où il n'y aurait ni békés, ni mulâtres, ni nègres, mais des hommes libres et égaux en droit" (45). Les traits de sa personnalité s'apparentent à ceux qu'Albert Memmi conféra au "colonisateur de bonne volonté" qui, "ayant découvert, et incapable d'oublier le scandale économique, politique et morale de la colonisation, il ne peut plus accepter de devenir ce que sont devenus ses compatriotes" (50). Ses croyances sont partagées par sa soeur Irmine dont la clairvoyance quant à la condition des Noirs est obscurcie par les stéréotypes que son éducation lui a inculqués. "Je voyais les Noirs partout subalternes, ombres soumises, [...] satisfaisant aux moindres caprices. [...] Je crois que leurs passions sont plus fortes que les nôtres et leurs rêves plus fous à l'intérieur de leurs têtes. Je crois qu'ils sont anarchiques. Parce qu'ils ont beaucoup souffert, ils sont susceptibles, agressifs, lents à se confier et à dire la vérité" (MC 108). La jeune de Linsseuil semble avoir bien assimilé la pensée de la Négritude qui selon Fanon oppose "la vieille Europe à la jeune Afrique, la raison ennuyeuse à la poésie, la logique oppressive à la piaffante nature, d'un côté raideur, cérémonie, protocole, scepticisme, de l'autre ingénuité, pétulance, liberté, pourquoi pas luxuriance. Mais aussi irresponsabilité" (DT 258). Le corps colonisé s'investit des caractéristiques qui sont traditionnellement attribuées au genre féminin et qui le confinent dans une position d'infériorité, ce qui fait apparaître le gouffre qui sépare les envahisseurs des envahis.

Ayant changé de camp en épousant Razyé, Irmine ne peut cependant pas être logée à la même enseigne que son frère. Celui-ci est détesté par ses anciens esclaves qui préféreraient qu'il ressemble plutôt à son père, ivrogne et cruel, pour qu'ils puissent le haïr sans avoir mauvaise conscience. Et cela puisque, comme le note Sandrine elle-même, "un maître, c'est un maître" (MC 195). Sa justice et sa bonté ne peuvent en effet être que relatives, vu qu'il fait toujours partie des privilégiés et qu'il profite des résultats du système colonial: "[...] il accepte que tout change [...] mais refuse d'envisager que cette révolution puisse entraîner un bouleversement de sa situation et de son être. Car c'est trop demander à l'imagination que d'imaginer sa propre fin, même si c'est pour renaître autre" (Memmi 70). Il n'est donc pas 
surprenant que ses plantations finissent par être brûlées à la suite d'un incendie annoncé déjà par Sandrine qui souhaite être un homme pour pouvoir participer à cet acte ultime de destruction.

Née le mois où on annonça l'abolition de l'esclavage, celle-ci, abandonnée par son père, resta avec sa mère au service des anciens colons et peina sous leur joug: "A cinq ans, je charroyais du bois. A sept ans, je fouaillais du linge sur les roches de la rivière Blanche. A dix ans, j'éventais le feu pour la cuisinière et tournais les sauces. A dix-huit ans, mes genoux dans l'eau savonneuse, je récurais les kilomètres de plancher de l'habitation" (MC 195). Son sort s'apparente à celui d'une autre mabo, Julie, qui raconte elle aussi ses misères: "J'ai satisfait les envies, celles du maître comme celles de ses amis ou de ses visiteurs. Parfois debout dans l'escalier. Ou couchée dans le galetas. J'ai calé l'estomac de mes bâtardes avec un peu de dictame ou de tolomane et j'ai gardé le bon lait qui moussait de mes seins pour les enfants de l'habitation" (114). Le corps de la femme noire devient le texte principal où s'écrit toute la violence coloniale. Instrument de servitude, objet de plaisir, il est dérobé de ses fonctions les plus intimes. Nourrice des enfants du Maître, Julie n'a plus de lait pour ses "bâtardes."

Comme le fait remarquer Joan Dayan: "Since slaves are construed as things without thought, then no amount of amputation, torture, or disfiguring can matter. [...] The master can deny the slave his body, owing him physically, affectively and nutritionally" (46-7). Considérée comme "un tas d'ordures" (MC 116), Julie sait bien qu'elle restera pour toujours une "négresse à Blancs" (115) et elle ne peut éprouver pour ses geôliers que "deuil, haine et ressentiment" (114). De même Sandrine est incapable de se débarrasser d'une haine qui "remue dans [s]on estomac, monte à [s]a bouche, fétide comme la bave d'un crapaud [...] [et qui] est sur le point de s'écouler en injures enfiévrées comme la lave du volcan, aiguisées comme la lame du couteau" (195). "Bouillonnante," pour reprendre les paroles d'Hélène Cixous, elle est prête à exploser, à faire éclater en d'innombrables petits morceaux cette image qu'on voulut qu'elle fasse sienne et qui était celle d'une femme "bien réglée, normale [...] d'un calme ... divin." C'est comme si sa rancune morale devenait corporelle, lui donnait une apparence "monstrueuse," dangereuse comme la lame d'un couteau, mais qui, pourrait l'aider à sortir de l'obscurantisme où elle était maintenue et à résister à la mort non seulement de son corps mais aussi de son âme (RM 40), ${ }^{5}$ mort qui la menace dans l'espace clos de l'habitation. Cette dernière est, comme nous le rappelle Cathy, hantée par la présence des femmes noires, mulâtresses et blanches qui y furent jadis les victimes les plus vulnérables. Durant la célébration de son union damnée avec Aymeric de Linsseuil, la jeune métisse est bien consciente qu'elle abandonne son enfance pour prendre "place de son plein gré dans une longue procession de victimes" (MC 57). Ses pensées qu'elle ne parvient pas à exprimer à haute voix sont formulées dans le récit de son frère Justin et font une fois de plus du domaine des BellesFeuilles un endroit où se concentre toute la brutalité coloniale et masculine:

Esclaves violées par des planteurs sadiques. Maîtresses empoisonnées pour des rivales 
et mourant dans des souffrances sans nom à la table des banquets. Vierges vendues pour de l'argent et des morceaux de terre à des vieux corps. Soeurs convoitées par leurs frères. Mères par leurs fils. Huit jours après ses noces, une épousée s'était jetée la tête en avant depuis la galerie circulaire du deuxième étage, et la tache de son sang colorait les pavés de l'entrée. (56-57)

Ce passé atroce reprend vie dans l'esprit de la nouvelle mariée et nous prépare déjà à son sort cruel ainsi qu'à celui d'autres personnages féminins à l'instar de sa fille qui meurt après une grossesse illicite ou bien d'Irmine dont le corps usé circule entre Razyé et Justin.

Tragiques, mais en même temps prometteurs, les parcours identitaires de ces femmes antillaises, mettent en branle toute une série de déconstructions qui ne sont pas effectuées sans obstacles, mais qui nous aident à défaire le texte-maître pour montrer ce qui est hors du système de représentation qu'il propose. Mutilée, par l'impérialisme colonial et patriarcal, la parole marginale, parfois faible, mais guérie de son mutisme, surgit pour rectifier celle des Maîtres et parvient ainsi à dénoncer ses failles et ses limites.

\section{Notes}

${ }^{1}$ La pensée de Bhabha se rapproche ici de celle de Gayatri Chakravorty Spivak quand elle souligne lors d'une de ses interviews: "I am critical of the binary opposition colonizer / colonized. I try to examine the heterogeneity of 'colonial power' and to disclose the complicity of the two poles of that opposition as it constitutes the disciplinary enclave of the critique of imperialism" (9). Voir, McRobbie, Angela. "Strategies of Vigilance: An Interview with Gayatri Chakravorty Spivak," Bloc 10 (1985): 5-9.

${ }^{2} \mathrm{LC}$ est l'abréviation de l'ouvrage critique de Chamoiseau, Patrick et Raphaël Confiant, Lettres créoles, cité en bibliographie.

${ }^{3} \mathrm{DT}$ est l'abréviation de l'ouvrage critique de Fanon, Frantz, Les Damnés de la terre, cité en bibliographie.

${ }^{4} \mathrm{MC}$ est l'abréviation du roman de Condé, Maryse La migration des coeurs, cité en bibliographie.

${ }^{5} \mathrm{RM}$ est l'abréviation de l'ouvrage critique de Cixous Hélène, "Le rire de la Méduse," cité en bibliographie. 


\section{Bibliographie}

Bhabha, Homi K. "Of Mimicry and Man. The Ambivalence of Colonial Discourse." October 28 (1984): 125-33.

. "The Other Question: Difference, Discrimination and The Discourse of Colonialism."

In Francis Baker, Peter Hulme, Margaret Iversen, Diana Loxley, eds, Literature, Politics and Theory. London and New-York: Methuen, 1986. 147-72.

Chamoiseau, Patrick et Raphaël Confiant. Lettres créoles. Paris: Hatier, 1991.

Cixous, Hélène. "Le rire de la Méduse." Arc 61 (1975): 39-54.

Condé, Maryse. La migration des coeurs. Paris: Robert Laffont, 1995.

Davies, Carole Boyce. "Private Selves and Public Spaces: Autobiography and the African Woman Writer." CLA 34.3 (1991): 267-89.

Dayan, Joan. "Codes of Law and Bodies of Color." Penser la créolité. Paris: Karthala, 1995. 41-67.

Detienne, Marcel et Jean-Pierre Vernant. Les ruses de l'intelligence: la métis des Grecs. Paris: Flammarion, 1970.

Durand, Gilbert. Les Structures anthropologiques de l'imaginaire. Paris: PUF, 1963.

Fanon, Frantz. Peau noire, masques blancs. Paris: Seuil, 1952. . Les Damnés de la terre. Paris: Ed. Maspéro, 1961.

Kristeva, Julia. Pouvoirs de l'horreur. Paris: Seuil, 1980.

Lejeune, Philippe. Le pacte autobiographique. Paris: Seuil, 1975.

Memmi, Albert. Portrait du colonisé. Paris: Payot, 1973.

Taguieff, Pierre-André. "Doctrines de la race et hantise du métissage. Fragments d'une histoire de la mixophobie savante." Nouvelle revue d'ethnopsychiatrie 17 (1991): 53100 . 